\title{
Force optimization of ionic polymer metal composite actuators by an orthogonal array method
}

\author{
YU Min ${ }^{1 \dagger^{* *}}$, HE QingSong ${ }^{1,2 \dagger}$, DING Yan $^{1}$, GUO DongJie ${ }^{1}$, LI JiaBo ${ }^{1} \&$ DAI ZhenDong ${ }^{1}$ \\ ${ }^{1}$ Institute of Bio-inspired Structure and Surface Engineering, Nanjing University of Aeronautics \& Astronautics, Nanjing 210016, China; \\ ${ }^{2}$ College of Mechanical and Electrical Engineering, Nanjing University of Aeronautics \& Astronautics, Nanjing 210016, China
}

Received January 4, 2011; accepted April 12, 2011

\begin{abstract}
Ionic polymer metal composites (IPMCs), a new kind of electro-active polymer, can be used for micro robotic actuators, artificial muscles and dynamic sensors. However, IPMC actuators have the major drawbacks of a low generative blocking force and dependence on a humid environment, which limit their further application. Multiple process parameters for the fabrication of IPMCs were optimized to produce a maximum blocking force; the parameters included reducing agent concentration, platinum salt concentration in the initial compositing process, and tetraethyl orthosilicate (TEOS) content. An orthogonal array method was designed and a series of fabrication experiments were carried out to identify the optimum process parameters. The results show that the platinum salt concentration in the initial compositing process plays the most significant role in improving the blocking force of IPMCs, the TEOS content plays an important role, and the reducing agent concentration has no apparent effect on the blocking force. In the optimized conditions, the IPMC actuator exhibited maximum blocking force of $50 \mathrm{mN}$, and the corresponding displacement was $14 \mathrm{~mm}$. Compared with normal conditions, the blocking force improved 2.4-fold without sacrificing the displacement, and the effective air-operating life was prolonged 5.8-fold for the blocking force and 5-fold for the displacement. This study lays a solid foundation for further applications of IPMCs.
\end{abstract}

ionic polymer metal composite, force optimization, orthogonal array method

Citation: Yu M, He Q S, Ding Y, et al. Force optimization of ionic polymer metal composite actua-tors by an orthogonal array method. Chinese Sci Bull, 2011, 56: 2061-2070, doi: 10.1007/s11434-011-4509-9

Actuation technology is an important symbol of the progress of mechanical systems. The actuator was developed for the steam engine, then internal combustion engines, and now for electromotors, making mechanical systems increasingly more flexible, with higher energy utilization and convenience [1]. New high-performance actuator materials that are capable of converting electrical energy to mechanical energy are needed for a wide range of demanding applications, such as micro-electromechanical systems (MEMS), micro air vehicles and disk drives [2]. Many studies focus on materials based on the piezoelectric effect and the cholesteric-nematic transition [3]. However, because of the

*Corresponding author (email: yumin@ @uaa.edu.cn)

$\dagger$ These authors contributed equally to the work high driving voltage, relatively small stroke, low frequency and large energy consumption, these materials may not satisfy the needs of microrobots and MEMS for high flexibility, redundancy rate and load/weight ratio. Thus the artificial muscle with low driving voltage and large deformation has experienced rapid growth in academic interest and industrial application $[4,5]$.

Since Oguro's group reported bending behavior in an applied electric field for the first time in 1992 [1], ionic polymer metal composites (IPMCs), also termed "artificial muscle", are considered to be one of the most promising EAPs (electroactive polymers), and have experienced rapid growth in academic interest and industrial application. An IPMC is composed of a perfluorinated ion exchange membrane (with ion exchange capacity $\sim 0.95 \mathrm{mmol} \mathrm{\textrm {H } ^ { + }} / \mathrm{g}$ poly- 
mer), which is chemically surface-composited with a conductive medium such as platinum or gold [6-8]. Typically, a strip of perfluorinated ionic polymer membrane that behaves like biological muscle bends toward the anode (in the case of cation exchange membranes) under the influence of an applied electric field, because of the migration of cations with water molecules in the ion exchangeable polymer membrane. Consequently, such IPMC strips oscillate in response to an alternating input voltage, and electrophoresis-like internal ion-water movement is responsible for creating effective strains for actuation [9-11]. Compared with piezoelectric ceramics, shape memory alloy and magnetostrictive material, IPMCs have several advantages such as a relatively large stroke with low driving voltage, light weight, low noise, bio-compatibility and low power consumption [12,13]. It is anticipated that they will be applied to soft robotic actuators and artificial muscles, as well as to dynamic sensors from the micro-to-macro size range [14]. Shahinpoor et al. employed IPMCs as actuators to fabricate a four-finger gripper [10]. Zhang et al. developed a new type of fish-like microrobot with swimming, walking and floating motions, which was actuated by ionic conducting polymer film (ICPF) actuators [15]. Yeom et al. designed a biomimetic jellyfish, which can be activated to mimic real locomotive behavior with pulse and recovery process. The vertical floating displacement and the thrust force of the biomimetic jellyfish robot under various input signals have been measured and compared [16]. However, IPMCs generate a small output force, which greatly limits their applications $[6,14]$, and there are increasing demands to improve the output force. Kim et al. adopted a solution recasting technique to fabricate three-dimensional IPMC artificial muscles. The test results showed that a successfully fabricated IPMC strip with $2 \mathrm{~mm}$ thickness produced generative forces of 20 gf under a small voltage [17]. Onishi et al. grew a suitable pair of gold electrodes with a fractal-like structure by sequential adsorption/reduction cycling for improvement of the deformation [18]. Lee et al. fabricated thick IPMC films by hot-pressing several thin IPMC films (5 pieces) and performed several cycles (4 plating cycles) of Pt electroless-plating to enhance the actuating performance (approximately 4 gf) [19]. Lee et al. employed multi-wall carbon nanotubes (MWCNT) as an additive to fabricate IPMCs with high electrical conductivity. The maximum blocking force found was $0.236 \mathrm{gf} / \mathrm{mm}^{2}$ [20].

The Pt electroding process of IPMCs is somewhat troublesome and time-consuming for achieving an identical $\mathrm{Pt}$ electrode layer. In the fabrication procedure of IPMCs there are many factors which intricately interact with each other, and precise control of the processing/fabrication parameters has a significant impact on the performance of IPMCs. The fabrication procedure is not the same for different research institutes, and needs further optimization and monitoring. We have successfully fabricated silica-perfluorosulfonic acid hybrid membrane in situ by sol-gel reaction, which increased the blocking force and prolonged the non-water working life of IPMCs [21]. Based on our previous investigation, in the present study we identify the parameters that significantly affect the performance of IPMCs, and employ an orthogonal array method to optimize the fabrication process and improve the blocking force of IPMCs, thus laying a solid foundation for the industrial applications of IPMCs.

\section{Experimental materials and methods}

\subsection{Experimental materials}

The perfluorosulfonic acid (PFSA) resin Nafion dispersion (DE-520) was obtained from DuPont Company. Poly(dimethylsiloxane) (PDMS; Sylgad 184) was purchased from Dow Corning. TEOS, $\mathrm{Pt}\left(\mathrm{NH}_{3}\right)_{4} \mathrm{Cl}_{2}, \mathrm{NH}_{4} \mathrm{OH}, \mathrm{NaBH}_{4}$, $\mathrm{H}_{2} \mathrm{NNH}_{2}$ and $\mathrm{H}_{2} \mathrm{NOH} \cdot \mathrm{HCl}$ were obtained from Aldrich. Other reagents were reagent grade and were used without further purification.

\subsection{Fabrication of IPMCs}

Starting from cast Nafion membrane, IPMCs were fabricated carefully by electroless plating [14,21]. The detailed steps were as follows. The first step (Nafion casting) was to prepare a mixed solution for recasting Nafion membrane. TEOS was added to the Nafion solution and stirred for $6 \mathrm{~h}$, then the mixed solution was poured into a rectangular container made from PDMS (30 $\mathrm{mm} \times 40 \mathrm{~mm} \times 50 \mathrm{~mm})$. After $20 \mathrm{~h}$ of evaporation at $70-90^{\circ} \mathrm{C}$, a silica hybrid membrane was formed; the membrane was then annealed at $150^{\circ} \mathrm{C}$ for 5 min. Hybrid Nafion $/ \mathrm{SiO}_{2}$ membranes were obtained with TEOS content $0.5,1$ and 1.5 wt. $\%$ and thickness $0.28,0.34$ and $0.42 \mathrm{~mm}$, respectively. A cast Nafion membrane with 0 wt.\% TEOS and thickness $0.22 \mathrm{~mm}$ was obtained by the same procedure. The second step (surface treatment) was to roughen the Nafion membrane surface to enable it to act as an effective electrode, by increasing the interfacial area between the electrode and the polymer electrolyte, as well as enhancing electroless plating. An anisotropic surface texture of the Nafion membrane was obtained by polishing with an abrasive material. The third step (ion adsorption) was to soak the ionic polymer in a salt solution to allow platinum-containing cations $\left(\left[\mathrm{Pt}\left(\mathrm{NH}_{3}\right)_{4}\right]^{2+}\right)$ to diffuse through via an ion-exchange process [22]. The fourth step (reduction) was to reduce the platinum complex cations adsorbed in the Nafion membrane to the metallic state in the form of nanoparticles, by using reducing agents such as $\mathrm{NaBH}_{4}$ [23]. The fifth step (developing) aimed to effectively grow Pt on top of the initial Pt surface to reduce the surface resistivity. The sixth step (ion exchange) was to introduce $\mathrm{Li}^{+}$ions into the IPMC sample by soaking it in an appropriate salt solution $(1.5 \mathrm{M} \mathrm{LiCl})$ at a moderate temperature (usually $30^{\circ} \mathrm{C}$ ) 
for 3 days.

\subsection{Design of optimized program}

There are many variables in the IPMC fabrication procedure. Based on our previous study, the fabricated IPMCs were optimized to produce maximum force by varying process parameters, in particular the concentration of reducing agent, platinum salt concentration in the initial compositing process, and the TEOS content. The orthogonal array method was used to identify the optimum process parameters. Using this method the number of experiments that were required to obtain optimization results in terms of force was significantly reduced. Three important factors were identified in the manufacturing process and four levels (low, center, high, higher) for each factor were chosen (Table 1). All three factors are low in the normal conditions. The orthogonal Table of $\mathrm{L}_{16}\left(4^{5}\right)$ (Table 2) was employed to design the test program. Table 2 shows the 16 combinations of factor

Table 1 Factors and relative levels

\begin{tabular}{ccccc}
\hline Factors levels & $\begin{array}{c}1 \\
\text { (low) }\end{array}$ & $\begin{array}{c}2 \\
\text { (center) }\end{array}$ & $\begin{array}{c}3 \\
\text { (high) }\end{array}$ & $\begin{array}{c}4 \\
\text { (higher) }\end{array}$ \\
\hline $\begin{array}{c}\text { Reducing agent concentration } \\
\text { A }(\%)\end{array}$ & 0.5 & 1.5 & 2.5 & 3.5 \\
$\begin{array}{c}\text { Platinum salt concentration } \\
\left.\text { B (mg mL }{ }^{-1}\right)\end{array}$ & 2 & 2.5 & 3 & 3.5 \\
$\begin{array}{c}\text { TEOS content } \\
\text { C }(\%)\end{array}$ & 0 & 0.5 & 1 & 1.5 \\
\hline
\end{tabular}

Table 2 Experimental design of an orthogonal array method

\begin{tabular}{|c|c|c|c|c|c|c|}
\hline $\begin{array}{l}\text { Test No. } \\
\text { |Factor }\end{array}$ & $1(\mathrm{~A})$ & 2(B) & $3(\mathrm{C})$ & $4(e)$ & $5(e)$ & $G_{i}(\mathrm{mN})$ \\
\hline 1 & 1 & 1 & 1 & 1 & 1 & 11.96 \\
\hline 2 & 1 & 2 & 2 & 2 & 2 & 25.97 \\
\hline 3 & 1 & 3 & 3 & 3 & 3 & 19.99 \\
\hline 4 & 1 & 4 & 4 & 4 & 4 & 32.05 \\
\hline 5 & 2 & 1 & 2 & 3 & 4 & 21.95 \\
\hline 6 & 2 & 2 & 1 & 4 & 3 & 19.99 \\
\hline 7 & 2 & 3 & 4 & 1 & 2 & 37.04 \\
\hline 8 & 2 & 4 & 3 & 2 & 1 & 35.97 \\
\hline 9 & 3 & 1 & 3 & 4 & 2 & 19.99 \\
\hline 10 & 3 & 2 & 4 & 3 & 1 & 32.05 \\
\hline 11 & 3 & 3 & 1 & 2 & 4 & 24.01 \\
\hline 12 & 3 & 4 & 2 & 1 & 3 & 33.03 \\
\hline 13 & 4 & 1 & 4 & 2 & 3 & 19.99 \\
\hline 14 & 4 & 2 & 3 & 1 & 4 & 25.97 \\
\hline 15 & 4 & 3 & 2 & 4 & 1 & 37.04 \\
\hline 16 & 4 & 4 & 1 & 3 & 2 & 28.03 \\
\hline$I_{j}$ & 89.97 & 73.89 & 83.99 & 108 & 117.02 & \\
\hline$I I_{j}$ & 114.95 & 103.98 & 117.99 & 105.94 & 111.03 & \\
\hline$I I I_{j}$ & 109.08 & 118.08 & 101.92 & 102.02 & 93.00 & \\
\hline$I V_{j}$ & 111.03 & 129.08 & 121.13 & 109.07 & 103.98 & \\
\hline$R_{j}^{2}$ & 45534.21 & 12117.44 & 46036.12 & 45191.63 & 45482.18 & \\
\hline$S_{j}=R_{j}^{2} / 4-C T$ & 92.90 & 428.37 & 218.37 & 7.25 & 79.89 & \\
\hline
\end{tabular}

levels that were used for optimization of the manufacturing process, to identify the procedure that gave maximum force. Columns 1, 2 and 3 represent factors A, B and C, respectively. The columns labeled " $e$ " were used for error estimation. $G_{i}$ is the blocking force of the IPMC manufactured in test $i . S_{j}$ is the sum of squares of deviations for each factor. $I_{j}, I I_{j}, I I I_{j}$ and $I V_{j}$ are the sums of $G_{i}$ with low, middle, high and higher level, respectively, in column $j . n$ is the total number of tests. The sum of squares of deviations of errors can be calculated from the blank columns 4 and 5 without factors, which reflect only the error in the tests.

\subsection{SEM observation}

Scanning electron microscopy (SEM) (LEO 1530VP) was used to observe the topographical structure of membrane cross-sections at $5.0 \mathrm{kV}$ accelerating voltage. The images were obtained using a Schottky field emission source, a beam booster maintaining high beam energy throughout the microscope column, an electromagnetic multi-hole beam aperture, and a magnetic field lens. Cross-sections of all samples were obtained by low temperature fracture. The Nafion and IPMC samples were fractured after immersion in liquid nitrogen for $30 \mathrm{~min}$. All samples were coated with Pt particles ( 20 nm) before SEM observation.

\subsection{Test apparatus for IPMC actuation}

The experimental setup comprised a signal generator, laser displacement sensor and force loadcell. Figure 1 shows the experimental setup. The IPMC test samples were driven by the signal generator (Sample, SP1651), which produced a sinusoidal voltage signal. The laser displacement sensor (Keyence, LK-G80) was placed so that the laser beam was perpendicular to the surface of the IPMC and projected a spot $16 \mathrm{~mm}$ from the fixed edge of the IPMC. The IPMC

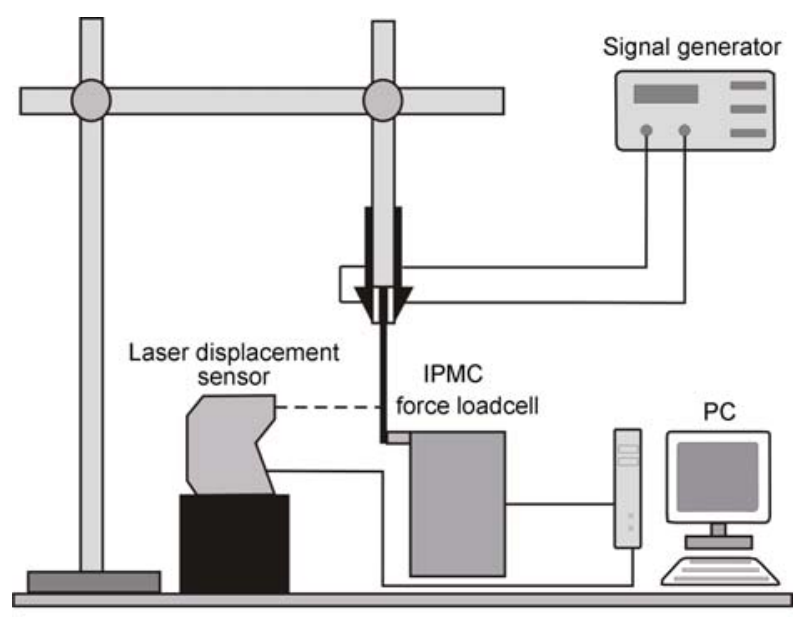

Figure 1 Test apparatus. 
test sample was attached at one end to the load cell (CETRUMT, $10 \mathrm{~g}$ ), which collected blocking force data.

IPMC samples were cut into strips with dimensions 20 $\mathrm{mm} \times 5 \mathrm{~mm}$ (length $\times$ width). Blocking force and displacement were measured in a $3 \mathrm{~V}$ AC electric field at $0.1 \mathrm{~Hz}$ frequency. One end of the IPMC strip was fixed between two $\mathrm{Cu}$ electrodes, and the other end was suspended vertically. All experiments were carried out in air at room temperature.

\section{Results and analysis}

\subsection{Variance analysis}

The maximum blocking force and displacement of IPMCs fabricated with the 16 different procedures were measured in the test apparatus (Figure 2). The results show that the IPMCs exhibited blocking force in the range 11.96-37.04 $\mathrm{mN}$, and displacements in the range 3.60-13.96 $\mathrm{mm}$. According to the measured maximum blocking force, the sum of the squares of the deviations of the factors and error were calculated as $S_{\mathrm{A}}=92.90, S_{\mathrm{B}}=428.37, S_{\mathrm{C}}=218.37$, $S_{e}=87.14$. The total degrees of freedom was $f_{\text {total }}=n-1=15$ ( $n$ is the number of tests), the degrees of freedom of the sum of the squares of the deviations of each factor $(\mathrm{A}, \mathrm{B}, \mathrm{C})$ was $f_{\mathrm{A}}=f_{\mathrm{B}}=f_{\mathrm{C}}=3$ (the number of levels -1 ), and the degrees of freedom of the sum of the squares of the deviations of the error $e$ was $f_{e}=f_{\text {total }}-f_{\mathrm{A}}-f_{\mathrm{B}}-f_{\mathrm{C}}=6$. The $F$ ratio is defined as the ratio of the average of the sum of the squares of the deviations of each factor to the average of the sum of the squares of the deviations of the error, and $F_{\mathrm{A}}=2.13, F_{\mathrm{B}}=9.83$, $F_{\mathrm{C}}=5.01$. The significance level can be obtained by comparing the $F$ ratio of every factor with the critical value. If the $F$ ratio is greater than the critical value, the difference in the test result can be attributed to the change in the levels. If not, the difference is attributed to experimental error. The critical value $F_{a}$ is determined according to the $F$ distribution table in statistics; $a$ is the reliability. The critical values were $F_{0.10}(3,6)=3.29, F_{0.05}(3,6)=4.76, F_{0.01}(3,6)=9.78$. Because $F_{\mathrm{A}}<F_{0.10}(3,6)$, the reducing agent concentration played no role in the blocking force of IPMCs. Because $F_{\mathrm{B}}>F_{0.01}(3,6)$, the platinum salt concentration had the most significant effect on the blocking force. Because

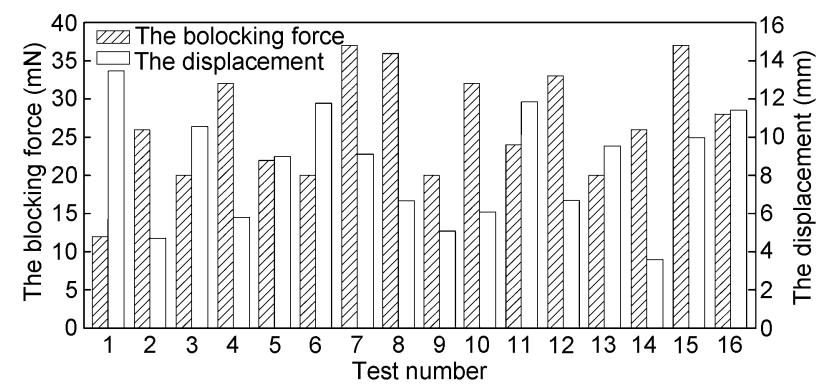

Figure 2 Maximum blocking force and displacement in the test program.
$F_{0.05}(3,6)<F_{\mathrm{C}}<F_{0.01}(3,6)$, the TEOS content had a significant effect on the blocking force of IPMCs. Table 3 shows the variance analysis of the orthogonal test. According to the calculated value of $I_{j}, I I_{j}, I I I_{j}$ and $I V_{j}$ (Table 2), $I I_{\mathrm{A}}$ exhibits the largest number (114.95), indicating that factor $\mathrm{A}$ should be at the center level. For the same reason, $I V_{\mathrm{B}}$ exhibits the largest value (129.08), indicating that factor B should be at the higher level, and $I V_{\mathrm{C}}$ exhibits the largest value (121.13), indicating that factor $C$ should be at the higher level. Thus it can be concluded from the variance analysis that the fabricated IPMCs can produce maximum force with the optimum parameters: reducing agent concentration $1.5 \%$ (center level); platinum salt concentration in the initial compositing process $3.5 \mathrm{mg} \mathrm{mL}$ (higher level); and TEOS content $1.5 \%$ (higher level).

\subsection{Blocking force and displacement}

By electroless plating, IPMCs were fabricated in the normal and optimal conditions; their performances for a working time of $800 \mathrm{~s}$ are shown in Figure 3. Figures 3(b) and 3(d) show the blocking force and displacement of IPMCs under the sinusoidal voltage. The forces in every period of the sinusoidal applied voltage were measured, and gave the curve of the relationship between maximum blocking force and working time in Figure 3(a). Similarly, the curve of the relationship between maximum displacement and working time was obtained (Figure 3(c)). For the IPMC actuator fabricated in normal conditions, the maximum blocking force and maximum displacement were $14.80 \mathrm{mN}$ and $13.96 \mathrm{~mm}$, respectively. For the IPMC actuator manufactured in optimal conditions the maximum blocking force and displacement were $49.98 \mathrm{mN}$ and $14.10 \mathrm{~mm}$, respectively. The maximum force of the IPMC fabricated in the optimal condition thus increased 2.4-fold without reducing the maximum displacement. Moreover, the maximum blocking force $(49.98 \mathrm{mN})$ and displacement $(14.10 \mathrm{~mm})$ were higher than the forces (11.96-37.04 $\mathrm{mN}$ ) and displacements $(3.60-13.96 \mathrm{~mm})$ of the IPMC samples in the sixteen experiments (Table 2).

According to the actuation mechanism of IPMCs, the

Table 3 Variance analysis ${ }^{\mathrm{a}}$

\begin{tabular}{cccccc}
\hline $\begin{array}{c}\text { Sources of } \\
\text { variance }\end{array}$ & $\begin{array}{c}\text { Sum of } \\
\text { square of } \\
\text { deviations }\end{array}$ & $\begin{array}{c}\text { Degrees } \\
\text { of } \\
\text { freedom }\end{array}$ & $\begin{array}{c}\text { Average of } \\
\text { the sum } \\
\text { of square } \\
\text { of } \\
\text { deviations }\end{array}$ & $F$ ratio Significane \\
\hline $\begin{array}{c}\text { Reducing agent } \\
\text { concentration (A) } \\
\text { Platinum salt }\end{array}$ & 92.90 & 3 & 30.97 & 2.13 & $/$ \\
$\begin{array}{c}\text { concentration (B) } \\
\text { TEOS content (C) }\end{array}$ & 218.37 & 3 & 72.79 & 5.01 & $*$ \\
Error $(e)$ & 87.14 & 6 & 14.52 & & $* *$ \\
\hline
\end{tabular}

a) "/" represents no apparent significance, $* *$ represents the greatest significance, and $*$ represents significance. 
performance should decline after working in air for a long time. In Figure 3(a) and 3(c) the performance durabilities of the IPMCs fabricated in the normal and optimal conditions are compared via the relationships between blocking force and working time, and between displacement and working time. For convenience we define the effective air-operating time of the IPMC as the actuating time until the performance (including force and displacement) of each specimen reached $80 \%$ of the maximum values. In the normal condition, the blocking force of the IPMC reached the maximum value $(14.80 \mathrm{mN})$ at $38.05 \mathrm{~s}$, then gradually decreased. It reached $80 \%$ of the maximum value $(11.76 \mathrm{mN})$ at $118.28 \mathrm{~s}$, and decayed to $6.57 \mathrm{mN}$ at $800 \mathrm{~s}$; the force thus decayed $60 \%$. In the optimal condition, the blocking force reached $39.78 \mathrm{mN}$ at $14.16 \mathrm{~s}$ and the maximum value $(49.98 \mathrm{mN})$ at $252.72 \mathrm{~s}$, and decreased gradually until the measurement was terminated. The IPMC exhibited a blocking force of $47.04 \mathrm{mN}$ after $800 \mathrm{~s}$, with only $6 \%$ decay. Thus, the blocking force output of the IPMC fabricated in the optimal condition was highly improved: the effective air-operating time was prolonged 5.8-fold. Similarly, in the normal condition, the displacement of the IPMC reached the maximum value $(13.96 \mathrm{~mm})$ initially, then rapidly decreased, and decayed to $80 \%$ of the maximum displacement in $52.8 \mathrm{~s}$ (Figure $3(\mathrm{c}))$. In the optimal condition, the IPMC main-
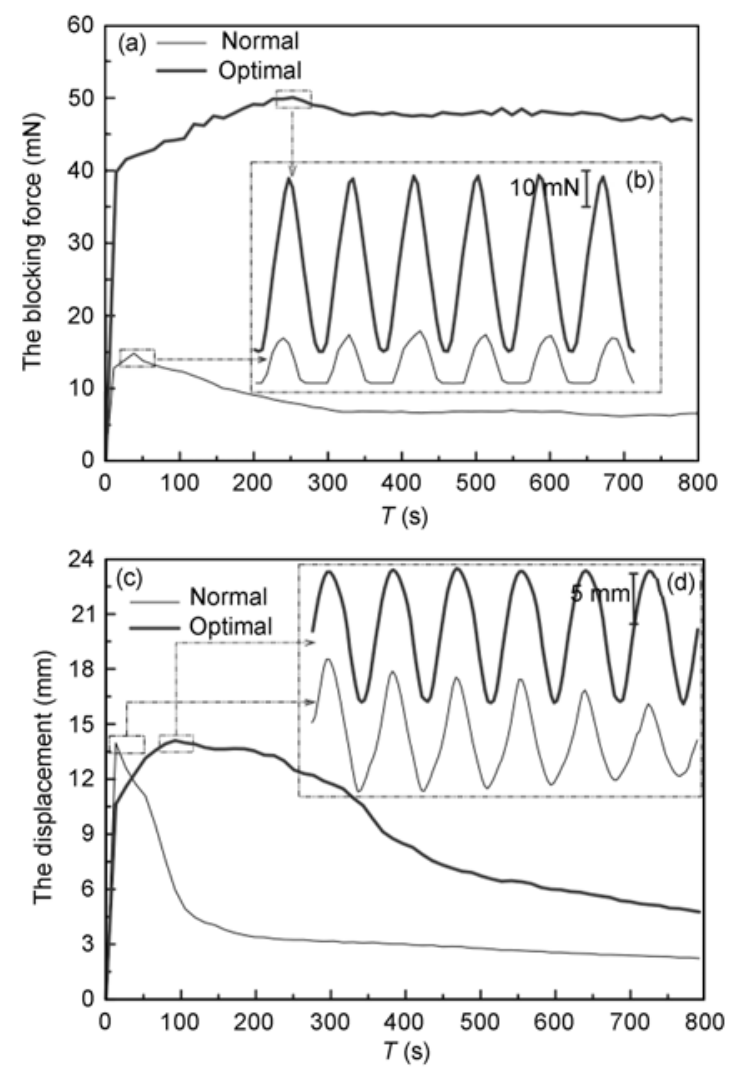

Figure 3 Blocking force and displacement of IPMCs versus time in the normal and optimal condition. (a) Force, and (b) maximum force, versus time; (c) displacement, and (d) maximum displacement; versus time. tained $80 \%$ of the maximum value after 316.8 s. These results show that the stability of the displacement output was substantially modified, with a 5-fold increase of the effective air-operating time.

The maximum, average and minimum of the force and displacement in the whole working time $(800 \mathrm{~s})$ are shown in Figure 4. The maximum reflects the peak work capability, while the average and minimum reflect steady operation capability. Whether the peak work capability or steady operation capability is considered, the blocking force of the IPMC fabricated in the optimal condition was greatly improved. At the same time, the steady operation capability of the displacement was enhanced without reduction of the maximum displacement.

\subsection{SEM observation}

Figure 5 shows cross-sectional SEM images of the IPMC samples (numbers 1, 2, 3 and 4) made with platinum salt concentrations $2,2.5,3$ and $3.5 \mathrm{mg} \mathrm{mL}^{-1}$. As the platinum salt concentration was increased, the thickness of plated platinum (the thickness of the surface electrode of the IPMC) increased from 6 to 7, 10 and $24 \mu \mathrm{m}$, from sample 1 to samples 2, 3 and 4, respectively. As noted previously, as the platinum penetration and the thickness of the metal electrode increase, the surface resistance decreases [18,19]. Thus, with the same input voltage the current increases,
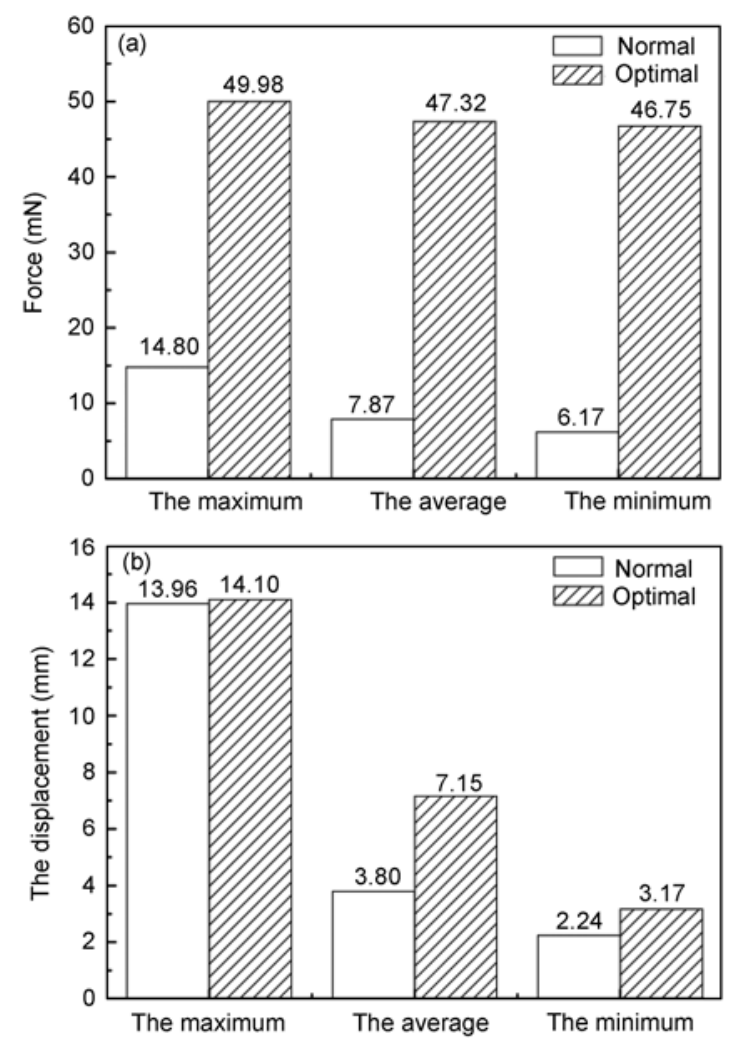

Figure 4 Performance of the IPMC in the optimal condition, compared with that in normal condition. (a) Force; (b) displacement. 

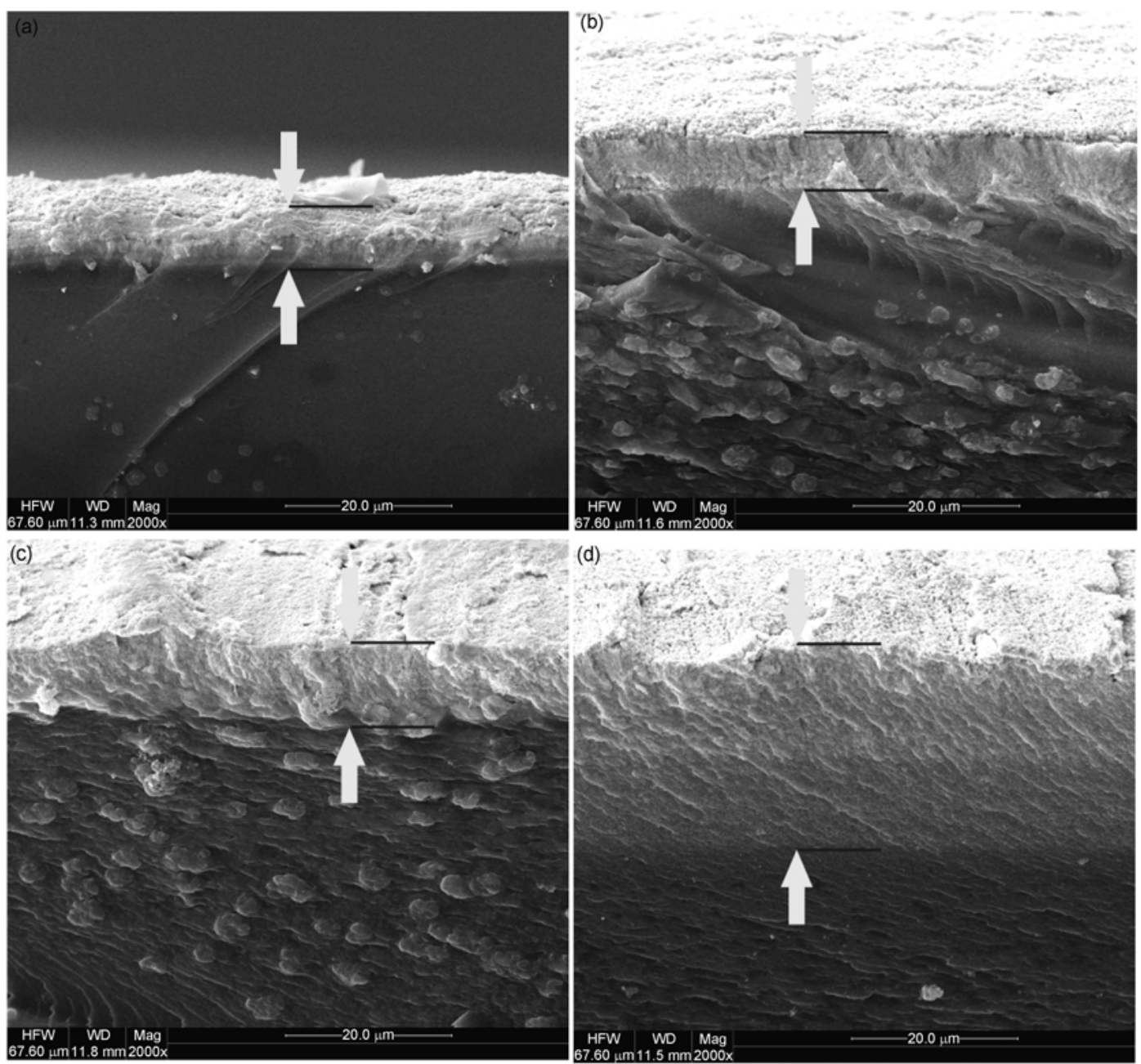

Figure 5 Cross-sectional SEM images of IPMC samples with platinum salt concentration. (a) 2; (b) 2.5; (c) 3 ; (d) $3.5 \mathrm{mg} \mathrm{mL}^{-1}$.

which greatly enhances the blocking force of the IPMC. Overall, it should be noted that IPMCs with low resistivity tend to produce larger blocking forces. When the platinum concentration was $3.5 \mathrm{mg} \mathrm{mL}^{-1}$ there was no visible interface between Pt grains and the Nafion membrane, indicating that $\mathrm{Pt}$ grains were deeply inserted inside the Nafion polymer. This IPMC sample had the thickest surface electrode $(\sim 24 \mu \mathrm{m})$, hence the smallest surface resistivity and largest blocking force. Figure 5(d) shows that the transition layer between the metal electrode and the polymer membrane was enlarged. Because of the compact and uniform connection, water movement near the electrode boundaries was restricted, thus providing good protection against water leakage, and benefitting the actuation behavior of the IPMC. According to the theory of electroless plating, the deposition velocity increases with increased platinum salt concentration. However, it decreases at very high concentration because of self-decomposition of the plating bath. Because the Nafion resin has limited ion exchange capacity, the amount of $\left[\mathrm{Pt}\left(\mathrm{NH}_{3}\right)_{4}\right]^{2+}$ that can be absorbed by the polymer is also restricted. Consequently, it is not necessary to use a very high platinum salt concentration in the initial compositing process.

This paper also reports a new technique for fabricating IPMC actuators. The technique is based on a hybrid organic-inorganic composite membrane, which can increase the ability of the membrane to retain water. In the fabrication procedure silica particles, prepared by in situ hydrolysis of TEOS by sol-gel reaction, co-crystallize with PFSA ionomer. When the TEOS content was $0.5,1$ or $1.5 \%$, amorphous silica co-crystallized with the PFSA macromolecule forming many micro scale channels inside the hybrid membrane. The silica ensures high water-retention capability, while the micro scale channels keep the water in reserve and act as water migrating conduits. Both the silica and the channels may optimize the actuation behavior of IPMCs. Figure 6(a) shows the smooth cross-section of PFSA membrane with few conduits. With the addition of TEOS, many micro scale channels appeared in the cross-section of the membrane. As the TEOS content increased from 0.5 to $1.5 \%$, the diameter of the channels increased to approximately 1,5 and $10 \mu \mathrm{m}$, respectively 

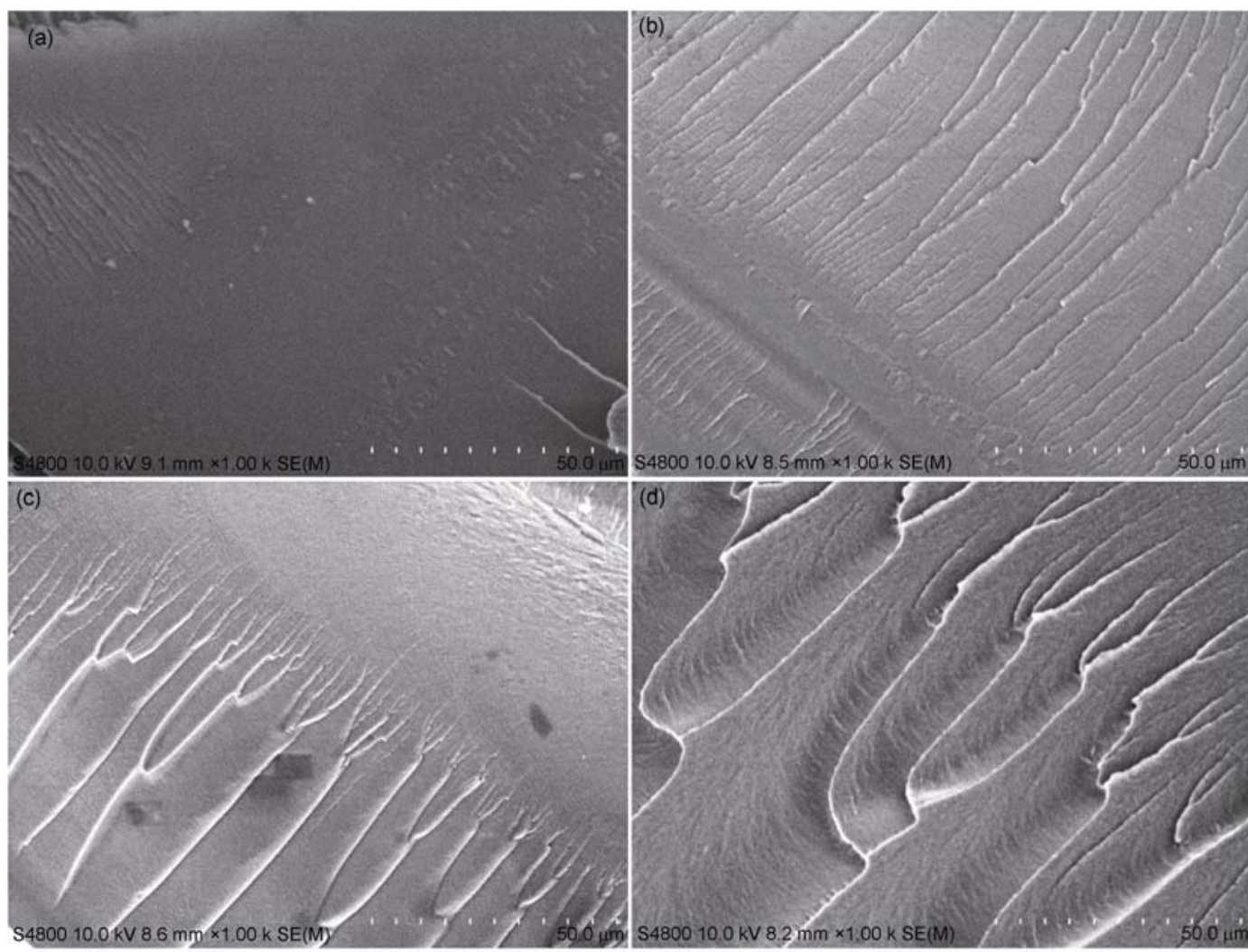

Figure 6 Cross-sectional SEM images of Nafion membrane with TEOS content. (a) 0 ; (b) $0.5 \%$; (c) $1 \%$; and (d) $1.5 \%$.

(Figures 6(b)-(d)), for $0.5,1.0$ and 1.5\% TEOS, respectively. There was a large number of micro scale channels inside the hybrid membrane, in addition to nano scale channels [21], thus forming a highly porous composite membrane. Both micro and nano scale channels act as water migrating conduits, and after fabrication into an IPMC actuator, those channels may be filled with water. When the TEOS content was increased the diameter of the micro channels increased, and the water storage capability consequently improved. The water storage capability can be characterized by the water content, $w$, which is expressed as follows:

$$
w=\frac{M_{\mathrm{wet}}-M_{\mathrm{dry}}}{M_{\mathrm{dry}}},
$$

where $M_{\text {wet }}$ and $M_{\text {dry }}$ are the weights of the Nafion membrane in the equilibrium swelling state and dry state, respectively, which were determined with an electronic balance (Mettler Toledo, AL204). When the TEOS contents were 0, $0.5 \%, 1 \%$ and $1.5 \%$, the water contents were $1.14 \%, 4.09 \%$, $6.53 \%$ and $15.27 \%$, respectively (Figure 7). Compared with the virgin Nafion membrane, the water content increased from 2.6- to $\sim 12.4$-fold (Figure 7 ) as the TEOS content increased. As the TEOS content of the membrane increased, and the diameter of the micro channels (Figure 6(b)-(d)) and water content increased, the quantity of hydrated cations migrating in the membrane increased at the same

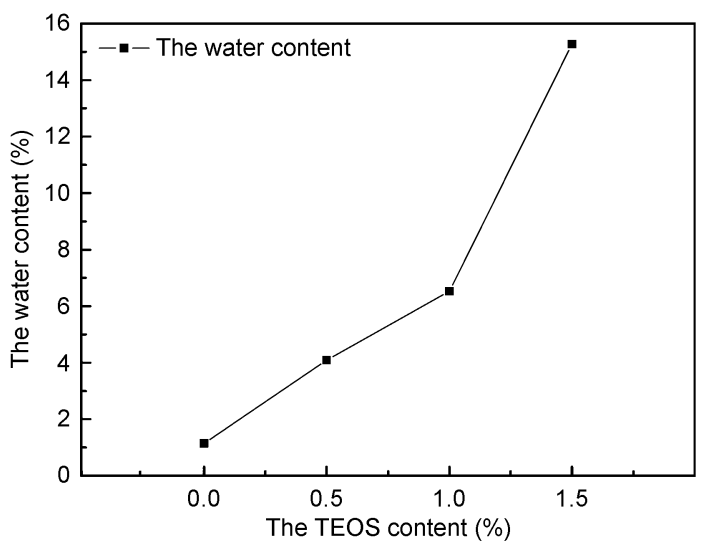

Figure 7 Water content of Nafion membranes as a function of TEOS content.

input signal. Consequently, a high water-retention PFSA membrane was obtained, which helped improve the effective air-operating life of the IPMC.

In summary, higher levels of factor B (platinum salt concentration=3.5 mg mL $\mathrm{m}^{-1}$ ) and factor $\mathrm{C}$ (TEOS content $=1.5 \%$ ) in the fabrication procedure play significant roles in enhancing the blocking force of the IPMC. The moderate platinum salt concentration is beneficial for forming a thicker and more compact metal electrode, which reduces the surface resistance and restricts water movement near the electrode boundaries. The large number of micro 
channels in the Nafion membranes incorporating TEOS resulted in increased water content, thus improving the maximum force and the steady operation capability of the membrane. It is clear that precise control of the fabrication procedure plays a key role in the actuation behavior of IPMCs.

\section{Discussion}

IPMCs can exhibit a large stroke under low driving voltage, but generate a relatively low blocking force. Hence there is increasing demand to improve the blocking force for further applications. In this section, three aspects are discussed to explain how the blocking force of IPMCs can be increased.

\subsection{Metal electrode}

Because of surface resistance, a voltage gradient is produced along the surface-electrode direction [7, 8]. Thus, the electric field inside the IPMC is not uniformly distributed, and the voltage at the electrode farther from the power supply is weaker. If the surface electrode of an IPMC has a large surface resistance, the voltage drop along the surface-electrode direction is appreciable, and the cations and solvent molecules inside the membrane will move toward the outer electrodes connected to the power supply, which greatly weakens the performance. Consequently it is necessary to reduce the surface resistance to decrease the voltage gradient as much as possible. When the surface resistance is low, the voltage applied through the outer electrodes will be almost constant over the whole surface electrode. The hydrated cations then mostly move up and down through the thickness, which is beneficial to the actuation performance of IPMCs. When the platinum salt concentration was increased from 2 to $3.5 \mathrm{mg} \mathrm{mL}^{-1}$, the thickness of the metal electrode increased from 6 to $24 \mu \mathrm{m}$ (Figure 5), and the surface resistance decreased 7.8 -fold from 7.8 to $1 \Omega \mathrm{cm}^{-1}$. A uniformly distributed platinum electrode was created, thereby implementing a granular damming effect, which slowed water movement near the electrode boundaries s and provided good protection against water leakage. Onishi et al. [18] and Lee et al. [19] have reported that the thickness of the surface metal electrode increased continuously with the number of plating steps by adsorption-reduction cycling, and that the surface resistance decreased. Although the thickness of the metal electrode was increased (to $20 \mu \mathrm{m}$ ), the reported fabrication procedure ( 8 plating cycles) is complicated and time-consuming. In the present work, an appropriate platinum salt concentration $\left(3.5 \mathrm{mg} \mathrm{mL}^{-1}\right)$ was determined by an orthogonal array method, and a metal electrode with thickness $24 \mu \mathrm{m}$ (Figure 5(d)) was formed in just 2 plating cycles, which optimized the fabrication procedure. The surface resistance and consumption of electrical energy of the IPMC were thus reduced, thereby increasing the electric field force of the hydrated cations and effectively enhancing the blocking force. Also, because of the granular damming effect water leakage near the electrode boundaries was reduced, and the effective air-operating life prolonged. The surface resistance can be reduced by manufacturing a composite electrode. For example, Shahinpoor et al. [7] showed that one way to solve the problem was to overlay a thin layer of a highly conductive metal (such as silver) on top of the platinum surface electrode. In the work reported by Lee et al. [8], gold was sputtered on the platinum electrode using PVD (physical vapor deposition) to improve the surface conductivity. In the present study, we found that the platinum salt concentration needed to reduce the surface resistance and form a good metal electrode has a significant role in synthesizing IPMCs.

\subsection{Hydration process}

Typically, the strip of perfluorinated ionic polymer membrane that behaves like a biological muscle bends toward the anode (in the case of cation exchange membranes) under the influence of an applied electric field, because of the migration of cations with water molecules in the ion exchangeable polymer membranes. Such IPMC strips oscillate in response to an alternating input voltage. Thus, electrophoresis-like internal ion-water movement is responsible for creating effective strains for actuation, and the bending capability of an IPMC is strongly related to the hydration of the associated cations [7, 24]. Cations with a higher hydration number should generate a higher blocking force and a larger displacement. However, because of electrolysis and leakage of water, the water is gradually lost and the water content and the hydration value in the membrane decrease. The ion channel through which hydrated cations move is then blocked, resulting in reduced actuation behavior. It follows that high water content helps improve the steady operation capability of an IPMC. Lee et al. [9] fabricated an IPMC containing a polypyrrole (PPy)/alumina composite filler to improve the water content and cation migration, which are attributed to the redox reaction of PPy and the hygroscopic effect of alumina. Compared with the bare Nafion IPMC, the maximum blocking force was doubled. Because ionic liquids have the advantage of greater electrochemical stability than water, thus offering the possibility of higher actuation voltages, the use of ionic liquids to replace water has been explored in many studies $[25,26]$. For instance, Bennett et al. [26] used 1-ethyl-3methylimidazolium trifluoromethanesulfonate ionic liquid to replace water in an IPMC. Experimental results indicated that the IPMC solvated with the ionic liquid had improved stability when operated in air, as compared to material solvated with water, oscillating $7 \times 10^{6}$ times in air with no degradation of performance. The main drawback associated with the use of ionic liquid is reduction in the speed of response $(<0.05 \mathrm{~Hz})$, which limits appli- 
cations. In the present work a perfluorosulfonic acid membrane with silicon dioxide was fabricated, significantly improving the water content and the hydration process. When the TEOS content was $1.5 \%$, the diameter of the micro channels in the membrane $(10 \mu \mathrm{m})$ and the water content $(15.27 \%)$ had their largest values. Thus, the hydration value was the highest and the quantity of hydrated cations migrating in the membrane was the highest, guaranteeing increased force and displacement and prolonging the effective air-operating life. Ionic liquid combined with water can be used as the solvent in future work.

\subsection{Electromechanical performance}

A number of interesting attempts to improve the blocking force and effective air-operating life have been reported. Lee et al. [9] employed PPy/alumina composite filler as an additive for fabricating a high performance IPMC actuator $(20 \times 5 \times 0.453 \mathrm{~mm})$. The artificial muscle exhibited maximum force per unit thickness of $18 \mathrm{mN} / \mathrm{mm}$ under a DC voltage of $3 \mathrm{~V}$. Nguyen et al. [27] used Nafion membranes modified with two kinds of silicate materials (fumed silica and montmorillonite) as the polyelectrolytes of IPMCs (20 $\mathrm{mm} \times 5 \mathrm{~mm} \times 0.3 \mathrm{~mm}$ ) to improve the performance. The maximum force per unit thickness was $52 \mathrm{mN} / \mathrm{mm}$ under DC voltage of $3 \mathrm{~V}$. Kim and Shahinpoor [17] obtained cast ion exchange membranes by carefully evaporating solvents from the solution. The test results showed that a successfully fabricated IPMC (length $\times$ width $\times$ thickness $20 \mathrm{~mm} \times 5$ $\mathrm{mm} \times 2 \mathrm{~mm}$ ) produced a generative blocking force of approximately 20 gf under a square-wave voltage of $6 \mathrm{~V}$ at frequency $0.5 \mathrm{~Hz}$. The maximum force per unit thickness was $100 \mathrm{mN} / \mathrm{mm}$. In the present work, an IPMC (20 mm× $5 \mathrm{~mm} \times 0.42 \mathrm{~mm}$ ) generated a blocking force of $49.98 \mathrm{mN}$ (Figure 6(a)) under a sinusoidal voltage of $3 \mathrm{~V}$ at $0.1 \mathrm{~Hz}$ frequency, and the force per unit thickness was $119 \mathrm{mN} / \mathrm{mm}$. By comparison it is apparent that the IPMC fabricated in the optimized conditions exhibited a greatly enhanced blocking force. Kim et al. [28] studied steady operation capability in a dry environment. For convenience they defined the effective air-operating lifetime of the actuator as the actuating time until the displacement reaches $60 \%$ of the maximum displacement. Their material decayed to $60 \%$ of the maximum displacement in about $200 \mathrm{~s}$. The displacement of the IPMC optimized in the present work reached $80 \%$ in 316.8 $\mathrm{s}$, and $60 \%$ of the maximum at $396 \mathrm{~s}$, which is twice the time reported by Kim et al. Thus, the steady operation capability of the optimized IPMC was improved to an impressive extent.

\section{Conclusions}

The fabricated IPMCs were optimized to produce maximum blocking force by varying three process parameters, namely the reducing agent concentration, platinum salt concentration in the initial compositing process and the TEOS content. IPMCs with increased blocking force and improved effective air-operating lifetime were obtained, and have great potential for application in underwater robotic actuators, MEMS and artificial muscles. The principal conclusions from this work are as follows.

(i) The platinum salt concentration in the initial compositing process (factor B) plays the most significant role in improving the blocking force of IPMCs, the TEOS content (factor C) plays a smaller but still important role, and the reducing agent concentration (factor $\mathrm{A}$ ) has no apparent effect.

(ii) The IPMC exhibits maximum blocking force $50 \mathrm{mN}$ and corresponding displacement $14.10 \mathrm{~mm}$ in the optimal condition, including the center level of factor $\mathrm{A}(1.5 \%)$, the higher level of factor $\mathrm{B}\left(3.5 \mathrm{mg} \mathrm{mL}^{-1}\right)$ and the higher level of factor $\mathrm{C}(1.5 \%)$. Compared with the IPMC in the normal condition, the maximum force increased 2.4-fold, and the effective air-operating lifetime in relation to output force and output displacement increased 5.8-fold and 5-fold, respectively. These improvements lay a solid foundation for further applications of IPMCs.

This work was supported by the National Natural Science Foundation of China (50705043, 50805076, 50975140).

1 NSFC Mechanics and Materials Science Department. Strategic Planning of Mechanical Engineering Discipline (2011-2020)(in Chinese). Beijing: Science Press, 2010

2 Aliev A E, Oh J, Kozlov M E, et al. Giant-stroke, superelastic carbon nanotube aerogel muscles. Science, 2009, 323: 1575-1578

3 Lehmann W, Skupin H, Tolksdorf C, et al. Giant lateral electrostriction in ferroelectric liquid-crystalline elastomers. Nature, 2001, 410: 447-450

4 Liu S, Liu Y, Cebeci H, et al. High electromechanical response of ionic polymer actuators with controlled-morphology aligned carbon nanotube/Nafion Nanocomposite electrodes. Adv Funct Mater, 2010, 20: 3266-3271

5 Bar-Cohen Y. Electroactive Polymer (EAP) Actuators as Artificial Muscles: Reality, Potential, and Challenges. Washington: SPIE Press, 2001

6 Kim K J, Shahinpoor M. Ionic polymer-metal composites: II. Manufacturing techniques. Smart Mater Struct, 2003, 12: 65-79

7 Shahinpoor M, Kim K J. The effect of surface electrode resistance on the performance of ionic polymer-metal composite (IPMC) artificial muscles. Smart Mater Struc, 2000, 9: 543-551

8 Lee S G, Park H C, Pandita S D, et al. Performance improvement of IPMC (Ionic Polymer Metal Composites) for a Flapping Actuator. Int J Con Aut Sys, 2006, 4: 748-755

9 Lee J W, Kim J H, Chun Y S, et al. The performance of Nafion-based IPMC actuators containing polypyrrole/alumina composite fillers. Macromol Res, 2009, 17: 1032-1038

10 Shahinpoor M, Kim K J. Ionic polymer-metal composites: IV. Industrial and medical applications. Smart Mater Struc, 2005, 14: 197-214

11 Park I S, Kim S M, Pugal D, et al. Visualization of the cation migration in ionic polymer-metal composite under an electric field. Appl Phys Lett, 2010, 96: 0433011-0433013

12 Shoji E, Hirayama D. The effects of the potential and polarization time on the performance of ionic polymer metal composite actuators: A control of forward and reverse displacements. Polym Adv Technol, 
2007, 18: 346-352

13 Tiwari R, Kim K J. Disc-shaped ionic polymer metal composites for use in mechano-electrical applications. Smart Mater Struct, 2010, 19: 0650161-0650167

$14 \mathrm{Yu} \mathrm{M}$, Shen $\mathrm{H}$, Dai Z D. Manufacture and performance of Ionic polymer-metal composites. J Bionic Eng, 2007, 4: 143-149

15 Zhang W, Guo S X, Asaka K. A New Type of Hybrid Fish-like Microrobot. Int J Autom Comp, 2006, 4: 358-365

16 Yeom S W, OH I K. A biomimetic jellyfish robot based on ionic polymer metal composite actuators. Smart Mater Struct, 2009, 18: 10

17 Kim K J, Shahinpoor M. A novel method manufacturing three-dimensional ionic polymer-metal composites (IPMC) biomimetic sensors, actuators and artificial muscles. Polymer, 2002, 43: 797-802

18 Onishi K, Sewa S, Asaka K, et al. Morphology of electrodes and bending response of the polymer electrolyte actuator. Electrochim Acta, 2000, 46: 737-743

19 Lee S J, Han M J, Kim S J, et al. A new fabrication method for IPMC actuators and application to artificial fingers. Smart Mater Struct, 2006, 15: 1217-1224

20 Lee D Y, Park I S, Lee M H, et al. Ionic polymer-metal composite bending actuator loaded with multi-walled carbon nanotubes. Sens Actuators A, 2007, 133: 117-127
21 Guo D J, Ding H T, Wei H J, et, al. Hybrids perfluorosulfonic acid ionomer and silicon oxide membrane for application in ion-exchange polymer-metal composite actuators. Sci China Ser E: Tech Sci, 2009, 52: 3061-3070

22 Millet P. Preparation of solid polymer electrolyte composites: Investigation of the ion-exchange process. J Appl Elec, 1995, 25: 227-232

23 Millet P. Preparation of solid polymer electrolyte composites: Investigation of the precipitation process. J Appl Elec, 1995, 25: 233239

24 Lee J H, Lee J H, Nam J D, et al. Water uptake and migration effects of electroactive ion-exchange polymer metal composite (IPMC) actuator. Sens Actuators A, 2005, 118: 98-106

25 Lee J W, Yoo Y T. Anion effects in imidazolium ionic liquids on the performance of IPMCs. Sens Actuators B, 2009, 137: 539-546

26 Bennett M D, Leo D J. Ionic liquids as stable solvents for ionic polymer transducers. Sens Actuators A, 2004, 115: 79-90

27 Nguyen V K, Lee J W, Yoo Y T. Characteristics and performance of ionic polymer-metal composite actuators based on Nafion/layered silicate and Nafion/silica nanocomposites. Sens Actuators B, 2007, 120: $529-537$

28 Kim S J, Lee I T, Kim Y H. Performance enhancement of IPMC actuator by plasma surface treatment. Smart Mater Struct, 2007, 16: N6-N11

Open Access This article is distributed under the terms of the Creative Commons Attribution License which permits any use, distribution, and reproduction in any medium, provided the original author(s) and source are credited. 